\title{
Dynamic alterations of the tongue in obstructive sleep apnea-hypopnea syndrome during sleep: analysis using ultrafast MRI
}

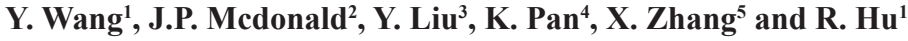 \\ ${ }^{1}$ Stomatology Hospital, Wenzhou Medical University, Wenzhou, \\ Zhejiang Province, China \\ ${ }^{2}$ Department of Orthodontics, Dental School, Glasgow University, \\ Glasgow, United Kingdom \\ ${ }^{3}$ Department of Orthodontics, Stomatology Hospital, Tongji University, \\ Shanghai, China \\ ${ }^{4}$ Department of Oral Surgery, Stomatology Hospital, Tongji University, \\ Shanghai, China \\ ${ }^{5}$ Sleep Center of the First Hospital, Wenzhou Medical University, \\ Wenzhou, Zhejiang Province, China
}

Corresponding author: R. Hu

E-mail: rongdanghu@126.com

Genet. Mol. Res. 13 (2): 4552-4563 (2014)

Received May 28, 2013

Accepted October 25, 2013

Published June 17, 2014

DOI http://dx.doi.org/10.4238/2014.June.17.7

\begin{abstract}
Patients with obstructive sleep apnea-hypopnea syndrome (OSAHS) were evaluated using ultrafast magnetic resonance imaging (UMRI) while asleep and awake to analyze tongue changes. The upper airway of 21 OSAHS patients and 20 normal controls were examined during sleep using UMRI. A series of midline sagittal images of the upper airway were obtained to measure dynamic changes in tongue size and the distance from the tongue to the $\mathrm{x}$-axis (an extended line from the anterior nasal spine to posterior nasal spine) and the y-axis (a perpendicular line from the center of the pituitary to the x-axis). The maximum and minimum sagittal
\end{abstract}


diameters of the tongue were shorter in the OSAHS group than in the control group $(\mathrm{P}<0.01)$ while awake, whereas the difference between the maximum and minimum vertical diameters of the tongue and the upper and central part of tongue between the posterior border and the retropharyngeal wall were greater $(\mathrm{P}<0.05)$. During sleep, the maximum values and differences between the maximum and minimum tongue sizes in the OSAHS group were larger than in the control group $(\mathrm{P}<0.05)$, whereas the minimum values were lower than in the control group $(\mathrm{P}<0.01)$. Tongue size significantly differs between OSAHS patients and normal controls during sleep. The tongue tends to move downward during OSAHS, which may be attributed to increased upper airway resistance.

Key words: Obstructive sleep apnea-hypopnea syndrome; Ultrafast magnetic resonance imaging; Upper airway resistance

\section{INTRODUCTION}

Obstructive sleep apnea-hypopnea syndrome (OSAHS) is characterized by repetitive obstruction of the upper airway during sleep (Okubo et al., 2006). Aside from obesity (Battagel et al., 2000; Dempsey et al., 2002), age (Hudgel et al., 2012), and gender (Shepertycky et al., 2005), craniofacial morphology is reportedly an important factor. Craniofacial morphologic abnormalities can be classified into skeletal anomalies such as small jaw, and soft tissue anomalies, such as enlargement of the soft palate or tongue area, which is related to the body mass index (BMI) (Battagel et al., 2000). Recent studies suggest that the consequences of craniofacial morphologic abnormalities are more severe in Asians than in Caucasians within a similar BMI range and degree of obesity (Li et al., 2000; Liu et al., 2000). Thus, craniofacial morphologic abnormalities should be considered during OSAHS risk assessment among Asian patients, which is based on the balance between the jaw and its associated soft tissue (Watanabe et al., 2005).

Many studies (Battagel et al., 2000; Schwab et al., 2003) that relate to skeletal and soft tissue morphology through cephalometry or magnetic resonance imaging (MRI) measure the lengths and angles of each variable specific to the method at the nasopharyngeal area, whereas a few studies have examined tongue shape and soft tissue alterations in the upper airway during sleep (Horiuchi et al., 2005; Patil et al., 2007). These morphologic alterations have been studied through various imaging techniques, including plain film cephalometrics, fluoroscopy, conventional CT, cine CT, conventional MRI, and MRI dynamic scans (Zhang et al., 2009). Considering that apnea and the respiratory cycle are dynamic processes, information on dynamic soft tissue alterations is necessary for analyzing the soft tissue morphology of OSAHS patients (Lowe et al., 1995; Trudo et al., 1998).

MRI is capable of showing soft tissue, which accurately reflects the actual anatomy of the upper airway of OSAHS patients. Ultrafast MRI (UMRI) is a recently developed technique that obtains images at multiple locations with sufficient quality and temporal resolution (less than $1 \mathrm{~s}$ ) to allow dynamic assessment of the upper airway and surrounding soft tissues (Ciscar et al., 2001; Rama et al., 2002; Pişkin et al., 2012). However, few studies have reported 
on the lingual shape in children with OSAHS using fast, dynamic MRI technology (Shott and Donnelly, 2004; Fricke et al., 2006; Zhang et al., 2009), and the tongue shape of adults with OSAHS has seldom been compared with normal subjects and during wakefulness and sleep (Lowe et al., 1995; Liu et al., 1999). Thus, in this study, dynamic alterations of the tongue during wakefulness and sleep were analyzed using UMRI to provide morphologic reference data related to the pathogenesis of OSAH.

\section{MATERIAL AND METHODS}

\section{Subjects}

Our study group consisted of 21 men (to avoid any potential influence of gender) with whom we consulted between February 2003 to May 2006, diagnosed with neutroclusion, and obtained consent to participate in the MRI study. All subjects were diagnosed with OSAHS using nocturnal polysomnography, with an average apnea-hypopnea index (AHI) of $20.30 \pm 9.46 / \mathrm{h}$. Each patient also underwent a thorough history (including the Epworth somnolence questionnaire) and a physical examination. These subjects had not received any treatment. Table 1 describes the characteristics of the study group. This study was conducted in accordance with the Declaration of Helsinki and with approval from the Ethics Committee of Wenzhou Medical University. Written informed consent was obtained from all participants.

Our control group consisted of 20 normal male subjects, with an average age of $45.62 \pm 7.35$ years, an average body weight of $70.47 \pm 4.64 \mathrm{~kg}$, an average body height of $169.21 \pm 3.38 \mathrm{~cm}$, and an average body mass index (BMI) of $24.48 \pm 3.87 \mathrm{~kg} / \mathrm{m}^{2}$. The control patients fulfilled the following requirements: 1) no snoring during sleep, based on reports from their partner; 2) no breathlessness or apnea during sleep at night; 3 ) no headache in the morning, nausea or daytime fatigue; 4) no obstructive pulmonary disease or pathology that might affect the upper airway; 5) no central pathology that might affect the workings of the respiratory center; and 6) no other relevant pathologic conditions.

\section{Polysomnography}

All patients underwent overnight polysomnography in a quiet dark room, using a 19-channel polygraph (Rembrandt Embla Polysomnography System, Monet 161 Medcare Flaga, Sidumuli 24, Reykjavik, Iceland). Sleep was documented using standard electroencephalographic (EEG), electro-oculographic (EOG), and electromyographic (EMG) criteria. The EEGs were recorded using electrodes applied to C3-A2 and C4-A1 (according to the criteria of Rechtschaffen and Kales) (Ruehland et al., 2011). EMG activity was recorded from the genioglossus, anterior tibial muscles, and diaphragmatic muscles. Apnea was defined as the cessation of airflow at the nose (two thermistors) and mouth (one thermistor) for longer than $10 \mathrm{~s}$. Hypopnea was defined as a 50\% reduction in airflow for 10 $\mathrm{s}$ associated with $\mathrm{a}>4 \%$ fall in oxygen saturation and/or an arousal. Apnea and hypopnea were considered to be obstructive when they occurred in the presence of thoracoabdominal movements. A single electrocardiogram (ECG) lead (modified V2) was monitored to detect cardiac arrhythmias. The polysomnograms were scored by a specialist in clinical neurophysiology. 


\section{Fixation and positioning of the heads of subjects}

Subjects were placed in a supine position with the orbitoauricular plane at a $95^{\circ}$ angle with the horizontal plane. A sponge pillow was placed under the cranial base on both lateral sides of the head. Quick-setting silicone rubber (Optosil Xantopren Comfort, Germany) was applied on the superficial coils of the MRI machine to affix the head in the interior of the neck coil after 3 min of coagulation. Airtight earplugs were inserted into the external auditory canal to minimize machine noise.

\section{Determination of sleep}

For the study group, sleepiness was documented when the four following situations occurred: 1) irregular snoring was heard through the microphones of the cylinder; 2) a decrease of $>4 \%$ in oxygen saturation was registered; 3 ) no sound was emitted by a rubber ball held by the patient and squeezed regularly; and 4) a slower and more disordered respiratory rhythm were monitored synchronously with respiratory-gated technology. Snoring and decreased oxygen saturation were observed only when patients were asleep.

For the control group, sleepiness was documented when the following situations occurred: 1) a decrease of $>4 \%$ in oxygen saturation was registered; 2 ) slowing and disruption of the respiratory rhythm were monitored synchronously using respiratory gated technology; and 3) no sound was emitted by the rubber ball being squeezed.

\section{Magnetic resonance imaging}

Subjects were placed in the mandibular rest position at resting respiration. The patients were examined at the Tenth Affiliated Hospital of Tongji University under continuous supervision by a radiologist and an orthodontist. The patients were deprived of sleep for $20 \mathrm{~h}$ prior to the MRI studies and were not allowed to take alcohol or sedatives on the day before the test. MRI scans were performed using a 1.5 superconducting magnet (Gyroscan Intera Type, Philips Co., Holland) in a posterior and anterior neck coil. The sequence used to perform the UMRI was a fast gradient-echo plus sequence. The technical parameters included the following: echo time, $12 \mathrm{~ms}$; repetition time, $2.3 \mathrm{~ms}$; flap angle, $25^{\circ}$; matrix, 192 x 512; and section thickness, $7 \mathrm{~mm}$. The imaging time per slice for this sequence was $0.92 \mathrm{~s}$. A total of 120 consecutive images were obtained as a single section, with a total imaging time of 110.4 $\mathrm{s}$. The MRI scans were first carried out with the subject asleep, and then while the subject was awake after 20 min of quiet respiration. All images were input into a PACS workstation.

\section{Tongue measurement}

The $\mathrm{x}$-axis represented an extended line from the anterior to the posterior nasal spine and the $y$-axis was a perpendicular line from the midpoint of the pituitary to the $\mathrm{x}$-axis (Figure 1A).

For the marker points, the posterior superior marginal point was designated as $\mathrm{A}$, the exterior edge of the cervical disc between the third and fourth cervical vertebrae was designated as B, the exterior edge of the cervical disc between the fourth and fifth cervical vertebra 
was designated as $\mathrm{C}$, the superior point of the mandible chin was designated as $\mathrm{D}$, and the fundus of the mandible was designated as E (Figure 1A).

For the test points, the superior margin of the tongue was designated as $\mathrm{H}$; the inferior margin of the tongue was designated as $\mathrm{L}$; the point intersected by a line parallel to the $\mathrm{x}$-axis from point $\mathrm{A}$ to the posterior border of tongue was designated as $\mathrm{A} 1$; the point intersected by a line parallel to the $\mathrm{x}$-axis from point $\mathrm{B}$ to the lingual posterior border was designated as $\mathrm{B} 1$; the point intersected by a line parallel to the $\mathrm{x}$-axis from the exterior edge of the cervical disc between the fourth and fifth cervical vertebrae (point $\mathrm{C}$ ) to the posterior lingual border was designated as $\mathrm{C} 1$; the point intersected by a line parallel to the $\mathrm{x}$-axis from point $\mathrm{D}$ to the posterior lingual border was designated as D1; and the point intersected by a line parallel to the $\mathrm{x}$-axis from point $\mathrm{E}$ to the posterior lingual border was designated as E1 (Figure 1B).
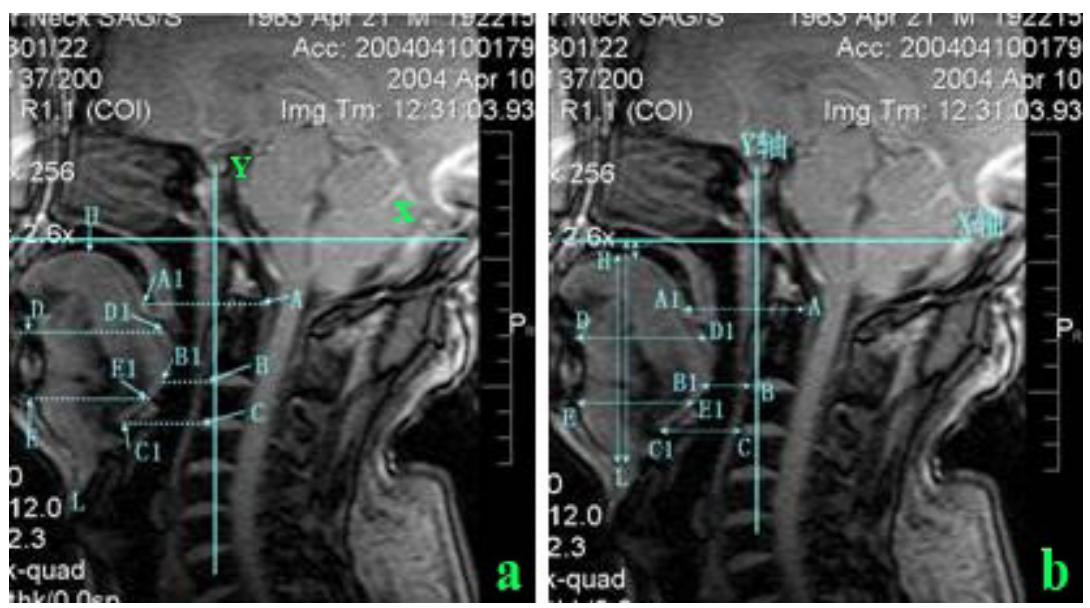

Figure 1. Coordinate and maker points for the measurement items of tongue. a. Coordinates: $x$ axis: an extended line from the anterior nasal spine to the posterior nasal spine; y axis: a perpendicular line from the mid-point of the pituitary to the $\mathrm{x}$ axis; Marker points: A. posterior superior marginal point of the pharyngeal cavity in the sagittal image; B) exterior edge of cervical disc between the third and fourth cervical vertebrae; C) exterior edge of cervical disc between the fourth and fifth cervical vertebrae; D) superior point of mandibular chin; E) fundus point of mandibular chin. b. Test points: H: superior margin of tongue; L: inferior margin of tongue; A1: point which was intersected by the parallel line with the $\mathrm{x}$ axis from point $\mathrm{A}$ to the posterior border of tongue; $\mathrm{B} 1$ : point intersected by the $\mathrm{x}$ axis parallel line from point $\mathrm{B}$ to the lingual posterior border; $\mathrm{C} 1$ : point intersected by the $\mathrm{x}$ axis parallel line from the exterior edge of cervical disc between the fourth and fifth cervical vertebrae (point $\mathrm{C}$ ) to the lingual posterior border; D1: point intersected by the $\mathrm{x}$ axis parallel line from point $\mathrm{D}$ to the lingual posterior border; E1: point intersected by the $\mathrm{x}$ axis parallel line from point $\mathrm{E}$ to the lingual posterior border.

\section{Dynamic alterations of the tongue size}

- D1-D represents the upper sagittal diameter of the tongue. The maximum and minimum D1-D and their difference were determined.

- E1-E represents the lower sagittal diameter of the tongue. The maximum and minimum E1-E, and their difference were determined.

- H-L represents the distance between the superior and the inferior lingual margins. Maximum and minimum values and their difference were determined. 


\section{Dynamic alterations in tongue position}

- A1-A: at the level of the superior lingual margin, the maximum and minimum distance from point $\mathrm{A} 1$ to the posterior lingual border and their difference were determined.

- B1-B: at the level of the cervical disc between the third and fourth cervical vertebrae, the maximum and minimum distance from point B1 to the posterior lingual border and their difference were determined.

- C1-C: at the level of the cervical disc between the fourth and fifth cervical vertebrae, the maximum and minimum distance between point $\mathrm{C} 1$ to the lingual posterior border and their difference were determined.

- $\mathrm{H}-\mathrm{X}$ : the maximum and minimum distance from point $\mathrm{H}$ of the tongue to the $\mathrm{x}$-axis and their difference were determined.

- L-X: the maximum and minimum distance from point $\mathrm{L}$ to the $\mathrm{x}$-axis and their difference were determined.

\section{Statistical analysis}

Pairs of means were statistically compared by $t$-tests using SPSS 10.1 for Windows (SPSS Inc, Chicago, IL, USA). Standard deviations of samples in both groups were evaluated using an F-test to confirm the homogeneity of variance before running the $t$-test. Differences with $\mathrm{P}<0.05$ were considered to be statistically significant.

\section{RESULTS}

\section{Clinical patient profiles}

The patient profile data in both study groups were statistically analyzed (Table 1). The subjects in the control group were approximately 5 years younger (mean, 45.6 years). The OSAHS group exhibited significantly higher BMIs than the control subjects. Correspondingly, the OSAHS subjects were shorter and had heavier body weights.

Table 1. Characteristics of the patients.
\begin{tabular}{lccc}
\hline Characteristics & OSAHS group (N $=21)$ & Control group $(\mathrm{N}=20)$ & Significance of differences $(t$-test) \\
& Mean \pm SD & Mean \pm SD & $* *$ \\
Age (years) & $50.79 \pm 9.75$ & $45.62 \pm 7.35$ & $* *$ \\
Body weight $(\mathrm{kg})$ & $74.77 \pm 7.63$ & $70.47 \pm 4.64$ & $* *$ \\
Body height $(\mathrm{cm})$ & $167.78 \pm 6.03$ & $169.21 \pm 3.38$ & $* *$ \\
BMI $\left(\mathrm{kg} / \mathrm{m}^{2}\right)$ & $29.30 \pm 4.55$ & $24.48 \pm 3.87$ & \\
\hline
\end{tabular}

After $\mathrm{F}$ test for the homegeneity test of variance, the data of OSAHS group was compared to the control group with independent samples $t$-test $(* * \mathrm{P}<0.01)$.

\section{Dynamic alterations in tongue size and position}

Dynamic alterations in tongue size and position in the normal group did not significantly differ between wakefulness and sleep for most parameters measured (Figure 2; Tables 2 and 3, rows a:b). Tongue size and the maximum D1-D value while asleep was higher 
than while awake, and the maximum E1-E value while asleep was lower than while awake $(\mathrm{P}<0.05$; Table 2, rows a:b). For tongue position, $\Delta \mathrm{L}-\mathrm{X}$ was higher while asleep than while awake $(\mathrm{P}<0.05$; Table 3, rows a:b). For the control group, data shown in Figure 2 reflect the remarkable similarity of tongue size and position whether the patient is awake or asleep.
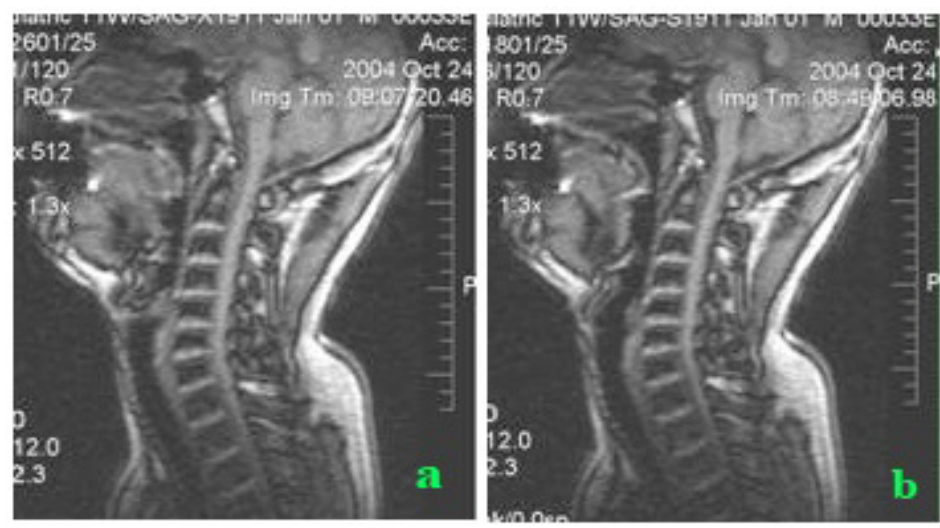

Figure 2. Tongue size and position for the normal group. a. Image of normal man whilst awake. b. Image of normal man whilst asleep; The dynamic alterations of the tongue size and position was not apparent between awake and asleep in the normal group.

Table 2. Comparison of tongue size between the obstructive sleep apnea-hypopnea syndrome (OSAHS) and normal group during awake and asleep.

\begin{tabular}{|c|c|c|c|c|c|c|c|c|c|}
\hline \multicolumn{2}{|c|}{ Tongue size items } & \multicolumn{2}{|c|}{ Normal $(\mathrm{N}=20)$} & \multicolumn{2}{|c|}{ OSAHS $(\mathrm{N}=21)$} & \multicolumn{4}{|c|}{ Significance of differences $(t$-test $)$} \\
\hline & & While awake (a) & In sleep (b) & While awake (c) & In sleep (d) & a:c & $a: b$ & $\mathrm{~b}: \mathrm{d}$ & $\mathrm{c}: \mathrm{d}$ \\
\hline \multirow[t]{3}{*}{$\overline{\mathrm{D} 1-\mathrm{D}}$} & Maximal value & $5.50 \pm 0.24$ & $5.68 \pm 0.26$ & $5.06 \pm 0.29$ & $5.44 \pm 0.33$ & $* *$ & * & $*$ & ** \\
\hline & Minimal value & $5.21 \pm 0.23$ & $5.35 \pm 0.30$ & $4.69 \pm 0.30$ & $4.39 \pm 0.31$ & ** & NS & $* *$ & ** \\
\hline & $\Delta \mathrm{D} 1-\mathrm{D}$ & $0.30 \pm 0.12$ & $0.33 \pm 0.11$ & $0.37 \pm 0.11$ & $1.05 \pm 0.16$ & NS & NS & $* *$ & ** \\
\hline \multirow[t]{3}{*}{ E1-E } & Maximal value & $4.90 \pm 0.89$ & $4.38 \pm 0.71$ & $4.73 \pm 0.39$ & $5.05 \pm 0.44$ & NS & $*$ & $* *$ & * \\
\hline & Minimal value & $4.62 \pm 0.98$ & $4.09 \pm 0.74$ & $4.40 \pm 0.47$ & $3.98 \pm 0.43$ & NS & NS & NS & ** \\
\hline & $\Delta \mathrm{E} 1-\mathrm{E}$ & $0.29 \pm 0.16$ & $0.30 \pm 0.20$ & $0.33 \pm 0.13$ & $1.07 \pm 0.19$ & NS & NS & $* *$ & ** \\
\hline \multirow[t]{3}{*}{ L-H } & Maximal value & $7.94 \pm 0.84$ & $8.05 \pm 0.68$ & $8.00 \pm 0.64$ & $8.43 \pm 0.62$ & NS & NS & * & ** \\
\hline & Minimal value & $7.70 \pm 0.86$ & $7.79 \pm 0.71$ & $7.67 \pm 0.66$ & $7.37 \pm 0.63$ & NS & NS & NS & NS \\
\hline & $\Delta \mathrm{L}-\mathrm{H}$ & $0.24 \pm 0.11$ & $0.28 \pm 0.12$ & $0.33 \pm 0.16$ & $1.07 \pm 0.21$ & $*$ & NS & $* *$ & $* *$ \\
\hline
\end{tabular}

$(* \mathrm{P}<0.05 ; * * \mathrm{P}<0.01)$. NS = no significance; $\Delta \mathrm{D} 1-\mathrm{D}=$ difference between maximal and minimal value of D1$\mathrm{D} ; \Delta \mathrm{E} 1-\mathrm{E}=$ difference between maximal and minimal value of $\mathrm{E} 1-\mathrm{E} ; \Delta \mathrm{L}-\mathrm{H}=$ difference between maximal and minimal value of $\mathrm{L}-\mathrm{H}$.

\section{Dynamic alterations in tongue size and position in the OSAHS group}

Rows c:d in Tables 2 and 3 show that most of the measured parameters, including the maximum and minimum values for tongue size and position and their differences ( $\Delta$ value), differed significantly between wakefulness and sleep. Unlike the control group, H-X, L-X, and H-L showed no statistically significant differences (Table 2 and 3, rows c:d). However, significant differences were observed for dynamic changes in tongue size and position in the OSAHS group between wakefulness and sleep (Figure 3). 
Table 3. Comparison of tongue position in the obstructive sleep apnea-hypopnea syndrome (OSAHS) and normal people when they are awake and asleep.

\begin{tabular}{|c|c|c|c|c|c|c|c|c|c|}
\hline \multicolumn{2}{|c|}{ Tongue position items } & \multicolumn{2}{|c|}{ Normal $(\mathrm{N}=20)$} & \multicolumn{2}{|c|}{ OSAHS $(\mathrm{N}=21)$} & \multicolumn{4}{|c|}{$\mathrm{t}$} \\
\hline & & While awake (a) & In sleep (b) & While awake (c) & In sleep (d) & $a: b$ & $\mathrm{a}: \mathrm{c}$ & $\mathrm{c}: \mathrm{d}$ & $\mathrm{b}: \mathrm{d}$ \\
\hline \multirow[t]{3}{*}{ A1-A } & Maximal value & $2.86 \pm 0.25$ & $2.95 \pm 0.29$ & $3.19 \pm 0.16$ & $3.55 \pm 0.21$ & NS & $* *$ & $* *$ & $* *$ \\
\hline & Minimal value & $2.43 \pm 0.24$ & $2.57 \pm 0.38$ & $2.54 \pm 0.20$ & $2.35 \pm 0.24$ & NS & NS & $* *$ & $*$ \\
\hline & $\Delta \mathrm{A} 1-\mathrm{A}$ & $0.43 \pm 0.19$ & $0.38 \pm 0.27$ & $0.44 \pm 0.12$ & $1.20 \pm 0.17$ & NS & NS & $* *$ & $* *$ \\
\hline \multirow[t]{3}{*}{ B1-B } & Maximal value & $1.70 \pm 0.38$ & $1.85 \pm 0.27$ & $1.92 \pm 0.29$ & $2.24 \pm 0.29$ & NS & $*$ & $* *$ & $* *$ \\
\hline & Minimal value & $1.46 \pm 0.37$ & $1.62 \pm 0.26$ & $1.59 \pm 0.33$ & $1.22 \pm 0.30$ & NS & NS & $* *$ & $* *$ \\
\hline & $\Delta \mathrm{B} 1-\mathrm{B}$ & $0.24 \pm 0.10$ & $0.23 \pm 0.10$ & $0.33 \pm 0.17$ & $1.02 \pm 0.17$ & NS & $*$ & $* *$ & $* *$ \\
\hline \multirow{3}{*}{$\mathrm{C} 1-\mathrm{C}$} & Maximal value & $2.63 \pm 0.25$ & $2.59 \pm 0.26$ & $2.79 \pm 0.56$ & $3.13 \pm 0.59$ & NS & NS & $*$ & $* *$ \\
\hline & Minimal value & $2.21 \pm 0.30$ & $2.25 \pm 0.29$ & $2.45 \pm 0.66$ & $1.93 \pm 0.63$ & NS & NS & $*$ & $*$ \\
\hline & $\Delta \mathrm{C} 1-\mathrm{C}$ & $0.42 \pm 0.15$ & $0.35 \pm 0.16$ & $0.35 \pm 0.14$ & $1.10 \pm 0.22$ & NS & NS & $* *$ & $* *$ \\
\hline \multirow[t]{3}{*}{$\mathrm{H}-\mathrm{X}$} & Maximal value & $0.29 \pm 0.07$ & $0.35 \pm 0.14$ & $0.55 \pm 0.18$ & $0.84 \pm 0.15$ & NS & $* *$ & $* *$ & $* *$ \\
\hline & Minimal value & $0.21 \pm 0.06$ & $0.22 \pm 0.08$ & $0.33 \pm 0.13$ & $0.23 \pm 0.06$ & NS & NS & NS & NS \\
\hline & $\Delta \mathrm{H}-\mathrm{X}$ & $0.08 \pm 0.08$ & $0.13 \pm 0.12$ & $0.21 \pm 0.10$ & $0.61 \pm 0.14$ & NS & $* *$ & $* *$ & $* *$ \\
\hline \multirow[t]{3}{*}{ L-X } & Maximal value & $7.94 \pm 0.84$ & $8.05 \pm 0.68$ & $8.57 \pm 0.76$ & $9.28 \pm 0.72$ & NS & NS & $*$ & $* *$ \\
\hline & Minimal value & $7.70 \pm 0.86$ & $7.99 \pm 0.71$ & $8.06 \pm 0.73$ & $7.93 \pm 0.73$ & NS & NS & NS & NS \\
\hline & $\Delta \mathrm{L}-\mathrm{X}$ & $0.24 \pm 0.11$ & $0.28 \pm 0.12$ & $0.52 \pm 0.19$ & $1.36 \pm 0.32$ & $*$ & $* *$ & $* *$ & $* *$ \\
\hline
\end{tabular}

$(* \mathrm{P}<0.05 ; * * \mathrm{P}<0.01) . \Delta \mathrm{A} 1-\mathrm{A}=$ difference between maximal and minimal value of $\mathrm{A} 1-\mathrm{A} ; \Delta \mathrm{B} 1-\mathrm{B}=$ difference between maximal and minimal value of B1-B; $\Delta \mathrm{C} 1-\mathrm{C}$ : difference between maximal and minimal value of $\mathrm{C} 1-\mathrm{C} ; \Delta \mathrm{H}-\mathrm{X}=$ difference between maximal and minimal value of $\mathrm{H}-\mathrm{X} ; \Delta \mathrm{L}-\mathrm{X}=$ difference between maximal and minimal value of $\mathrm{L}-\mathrm{X}$.
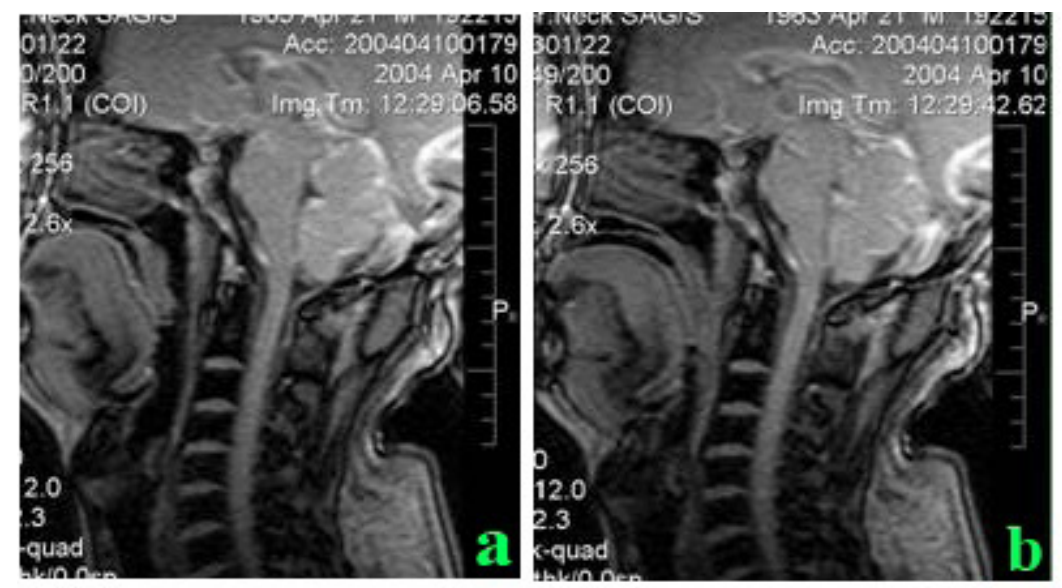

Figure 3. Tongue size and position for the OSAHS group. a. Image of OSAHS man whilst awake. The sagittal diameter of the superior part of the tongue is relatively small, the distance to the posterior pharyngeal wall is large while the distance to the hard palate is small for awake in OSAHS patients. b. Image of OSAHS man whilst asleep. The sagittal diameter of the superior part of the tongue is relatively large; the distance to the posterior pharyngeal is small for asleep OSAHS patients. The dynamic alterations of the tongue size and position were more apparent between awake and asleep in OSAHS patients.

\section{Comparison of tongue size and position between the OSAHS cohort and control group while awake}

Significant differences were observed between the OSAHS cohort and control group while awake, such as for the maximum and minimum D1-D, $\Delta \mathrm{L}-\mathrm{H}, \Delta \mathrm{H}-\mathrm{X}$, and $\Delta \mathrm{L}-\mathrm{X}$ parameters (Tables 2 and 3, rows a:c). Compared with the maximum and the minimum D1-D values 
in the normal controls, those in the OSAHS group were lower $(\mathrm{P}<0.01$; Table 2 , rows a:c $)$, whereas the maximum distance between the posterior pharynx and the posterior lingual border was larger, especially in the upper part of pharynx (A1-A) and the middle part of pharynx $(\mathrm{P}<0.05$; Table 3, rows a:c), as was the maximum $\mathrm{H}-\mathrm{X}$ value and the differences between the maximum and minimum $\mathrm{H}-\mathrm{X}$ and $\mathrm{L}-\mathrm{X}$ values $(\mathrm{P}<0.01$; Table 3 , rows a:c).

\section{Comparison of tongue size and position between the OSAHS cohort and the con- trol group while asleep}

Compared with normal adults, the maximum and the changes in each component of tongue position and size were significantly higher among OSAHS patients while asleep $(\mathrm{P}<0.05)$, whereas the minimum of each component of tongue position and size were smaller among the OSAHS patients $(\mathrm{P}<0.01)$. These findings suggest that larger alterations in tongue size and position occur among sleeping OSAHS patients compared with normal males (Tables 2 and 3 , rows b:d).

\section{DISCUSSION}

OSAHS is a multifactorial disorder that is influenced by factors such as obesity (Dempsey et al., 2002), age (Hudgel et al., 2012), and gender (Shepertycky et al., 2005). Craniofacial morphology, including skeletal and soft tissue morphology, is also an important factor that contributes to upper airway collapse during sleep (Takai et al., 2012). Considering that patients with OSAHS have apneic episodes and louder snores, researchers (Isono et al., 2003) hypothesized that variations in tongue size and position play an important role in OSAHS severity.

Previous methods used to study tongue morphology and movements include MRI (Shott and Donnelly, 2004; Fricke et al., 2006) and radiation techniques. Trudo et al. (1998) studied the size of the upper airways in normal adults using SE sequence MRI during sleep and found that tongue inclination increased. Yoshida et al. (1999) investigated the tongue retroposition phenomenon in OSAHS patients using low matrix MRI without statistical measurements. Donnelly et al. (2000) observed 70 young OSAHS patients using radiation techniques and found that $24 \%$ had tongue glossoptosis. Shott and Donnelly (2004) observed 15 young patients with Down's syndrome after tonsillectomy with persistent upper airway obstruction and found that $80 \%$ exhibited tongue glossoptosis. Tsuiki et al. (2008) reported that OSAHS patients have significantly larger tongues for a given maxillomandible size than non-OSAHS subjects. However, because of technical limitations, dynamic changes in tongue shape and position during sleep are seldom reported (Shott and Donnelly, 2004; Fricke et al., 2006; Zhang et al., 2009). In this study, we applied UMRI, which obtained images within less than $1 \mathrm{~s}$, to assess tongue measurements dynamically and to compare tongue size and position between the OSAHS patients and normal adults while awake or asleep.

Certain craniofacial structures, such as retrognathia and micrognathia, have been shown to predispose patients to OSAHS. In this study, all subjects received dental examinations and only males with Class I occlusions were selected to reduce possible deviations in skeletal morphologic abnormalities and gender. Consequently, the OSAHS subjects demonstrated higher BMIs, heavier body weights, older ages, and shorter height (Table 1). This ten- 
dency coincides with previous reports (Dempsey et al., 2002; Hudgel et al., 2012) that suggest that local soft tissue alterations in OSAHS patients might be partial manifestations of general processes such as obesity and aging.

To obtain an overview of tongue alterations in patients with OSAHS, we focused on changes in tongue size and position. Considering the inconsistency of tongue morphology and the uncertainty of the marker points, we referenced the tongue measurement method reported by Pae and Lowe (1999) and chose three reproducible lines to represent tongue size, two of which represent the tongue sagittal diameter and another that represents the vertical diameter. In addition, to reflect the dynamic tongue alterations, we chose four reproducible lines to represent the movement of the tongue surface at the posterior border (Figure 1).

The patency of the upper airway in humans is mainly maintained by muscle activation, wherein the genioglossus muscle reportedly plays a major role in normal adults during sleep (Worsnop et al., 1998). The maximum E1-E values, which represent the tongue sagittal diameter at the lower level, were lower in sleeping subjects $(\mathrm{P}<0.05)$, which suggests shrinkage of the genioglossal muscle. $\Delta \mathrm{L}-\mathrm{X}$, which represents the change in distance between the $\mathrm{x}$-axis and the inferior extremity, was greater in sleeping subjects $(\mathrm{P}<0.05)$, which suggests that the tongue has a large range of vertical movement. Our results agree with those reported by Trudo et al. (1998) and Worsnop et al. (1998).

The maximum and minimum values, as well as the changes in tongue size and position, were increased in sleeping subjects (Tables 2 and 3, rows a:b; $\mathrm{P}<0.01$ ), which reflect the considerable compliance of the tongue. The minimum A1-A, B1-B, C1-C, D1-D, and E1-E measurements, which represent tongue size and position in the sagittal direction, exhibited statistically significant differences $(\mathrm{P}<0.01)$ while asleep. The minimum $\mathrm{H}-\mathrm{X}, \mathrm{L}-\mathrm{X}$, and L-H values, which represent vertical tongue size and position, showed no statistically significant differences, which suggest that tongue size and position changed more in the sagittal direction than the vertical.

Our data indicate that significant differences exist between normal adults and OSAHS patients for tongue position and size, especially during sleep. The control adults had greater maximum D1-D values, which represent the sagittal diameter of the upper part of tongue during sleep, suggesting that the upper part of the tongue becomes more relaxed during sleep, even in normal adults. However, the OSAHS patients had more significant changes in both tongue size and dynamic changes during sleep (Tables 2 and 3), which indicate that the tongues of OSAHS patients have a large dynamic range of motion in both the vertical and horizontal planes during sleep.

We could not confirm previously reported data for craniofacial morphology; however, our results are consistent with previous studies on muscle function (Fogel et al., 2001; Younes et al., 2012). In the OSAHS group with an anatomically narrow and collapsible upper airways, the patients needed to inhale more vigorously to gain sufficient ventilatory capacity; hence, the pharyngeal sphincter muscle activation increased to keep the upper airway open. This compensatory mechanism was also confirmed by clinical trials (Mezzanotte et al., 1992), which indicated the absence of such a compensatory effect in normal subjects during apnea.

Tongue size and range of motion also showed statistically significant variations in the vertical direction. These findings suggest a mechanism in which the negative pressure in the upper airway decreases with the secondary deep inspiration following airway obstruction, causing the tongue to move downward. This could partly stimulate the genioglossus to shrink 
and cause the upper airway to reopen with the increased intrapulmonary pressure caused by hypoxemia. The shrinkage of the genioglossus reduces the tongue vertical diameter to move upwards in response to the expiratory flow. Therefore, the lingual range of motion can also be increased in the vertical direction.

This study verifies that the loss of the compensatory mechanism of genioglossus muscle activation and the weak response to negative pressure causes increased compliance of the tongue and consequential upper airway obstruction. These findings suggest new methods for treating OSAHS.

Although we limited the size of our patient cohort to reduce the influence of gender and skeletal craniofacial abnormalities, the etiology of sleep breathing disorders is multi-factorial and alterations in tongue size and position are only part of the equation. In addition, the altered pathophysiology of the airway under these conditions must also be recognized.

\section{ACKNOWLEDGMENTS}

Research partially supported by the Cooperation and Exchange projects of Wenzhou City Technology Bureau (Grant \#H2008002) and The Zhejiang Provincial Medical and Health Technology Fund (Grant \#2012KYA127). The authors would like to acknowledge the Tenth Hospital Affiliated to Tongji University for providing the ultrafast magnetic resonance imaging instrument to acquire a series of midline sagittal images of the upper airway of patients. Trial Registration: ClinicalTrials.gov, \#NCT01687855.

\section{REFERENCES}

Battagel JM, Johal A and Kotecha B (2000). A cephalometric comparison of subjects with snoring and obstructive sleep apnea. Eur. J. Orthod. 22: 353-365.

Ciscar MA, Juan G, Martinez V, Ramon M, et al. (2001). Magnetic resonance imaging of the pharynx in OSA patients and healthy subjects. Eur. Respir. J. 17: 79-86.

Dempsey JA, Skatrud JB, Jacques AJ, Ewanowski SJ, et al. (2002). Anatomic determinants of sleep-disordered breathing across the spectrum of clinical and nonclinical male subjects. Chest 122: 840-851.

Donnelly LF, Strife JL and Myer CM, III (2000). Glossoptosis (posterior displacement of the tongue) during sleep: a frequent cause of sleep apnea in pediatric patients referred for dynamic sleep fluoroscopy. AJR Am. J. Roentgenol. 175: $1557-1560$.

Fogel RB, Malhotra A, Pillar G, Edwards JK, et al. (2001). Genioglossal activation in patients with obstructive sleep apnea versus control subjects. Mechanisms of muscle control. Am. J. Respir. Crit. Care Med. 164: 2025-2030.

Fricke BL, Donnelly LF, Shott SR, Kalra M, et al. (2006). Comparison of lingual tonsil size as depicted on MR imaging between children with obstructive sleep apnea despite previous tonsillectomy and adenoidectomy and normal controls. Pediatr. Radiol. 36: 518-523.

Horiuchi A, Suzuki M, Ookubo M, Ikeda K, et al. (2005). Measurement techniques predicting the effectiveness of an oral appliance for obstructive sleep apnea hypopnea syndrome. Angle Orthod. 75: 1003-1011.

Hudgel DW, Lamerato LE, Jacobsen GR and Drake CL (2012). Assessment of multiple health risks in a single obstructive sleep apnea population. J. Clin. Sleep Med. 8: 9-18.

Isono S, Tanaka A and Nishino T (2003). Dynamic interaction between the tongue and soft palate during obstructive apnea in anesthetized patients with sleep-disordered breathing. J. Appl. Physiol. 95: 2257-2264.

Li KK, Kushida C, Powell NB, Riley RW, et al. (2000). Obstructive sleep apnea syndrome: a comparison between FarEast Asian and white men. Laryngoscope 110: 1689-1693.

Liu WJ, Cao WQ, Meng GM, Ge RF, et al. (1999). Study on pharyngeal morphology and its clinical significance. Chin. J. Clin. Anatomy 01: 1.

Liu Y, Lowe AA, Zeng X, Fu M, et al. (2000). Cephalometric comparisons between Chinese and Caucasian patients with obstructive sleep apnea. Am. J. Orthod. Dentofacial Orthop. 117: 479-485. 
Lowe AA, Fleetham JA, Adachi S and Ryan CF (1995). Cephalometric and computed tomographic predictors of obstructive sleep apnea severity. Am. J. Orthod. Dentofacial Orthop. 107: 589-595.

Mezzanotte WS, Tangel DJ and White DP (1992). Waking genioglossal electromyogram in sleep apnea patients versus normal controls (a neuromuscular compensatory mechanism). J. Clin. Invest. 89: 1571-1579.

Okubo M, Suzuki M, Horiuchi A, Okabe S, et al. (2006). Morphologic analyses of mandible and upper airway soft tissue by MRI of patients with obstructive sleep apnea hypopnea syndrome. Sleep 29: 909-915.

Pae EK and Lowe AA (1999). Tongue shape in obstructive sleep apnea patients. Angle Orthod. 69: 147-150.

Patil SP, Schneider H, Schwartz AR and Smith PL (2007). Adult obstructive sleep apnea: pathophysiology and diagnosis. Chest 132: 325-337.

Pişkin B, Sipahi C, Köse M, Karakoç Ö, et al. (2012). Use of an individual mandibular advancement device for an obstructive sleep apnea patient with facial paralysis: a short-term follow-up case report. J. Oral Rehabil. 39: 472-478.

Rama AN, Tekwani SH and Kushida CA (2002). Sites of obstruction in obstructive sleep apnea. Chest 122: 1139-1147.

Ruehland WR, O'Donoghue FJ, Pierce RJ, Thornton AT, et al. (2011). The 2007 AASM recommendations for EEG electrode placement in polysomnography: impact on sleep and cortical arousal scoring. Sleep 34: 73-81.

Schwab RJ, Pasirstein M, Pierson R, Mackley A, et al. (2003). Identification of upper airway anatomic risk factors for obstructive sleep apnea with volumetric magnetic resonance imaging. Am. J. Respir. Crit. Care Med. 168: 522-530.

Shepertycky MR, Banno K and Kryger MH (2005). Differences between men and women in the clinical presentation of patients diagnosed with obstructive sleep apnea syndrome. Sleep 28: 309-314.

Shott SR and Donnelly LF (2004). Cine magnetic resonance imaging: evaluation of persistent airway obstruction after tonsil and adenoidectomy in children with Down syndrome. Laryngoscope 114: 1724-1729.

Takai Y, Yamashiro Y, Satoh D, Isobe K, et al. (2012). Cephalometric assessment of craniofacial morphology in Japanese male patients with obstructive sleep apnea-hypopnea syndrome. Sleep Biol. Rhythms 10: 162-168.

Trudo FJ, Gefter WB, Welch KC, Gupta KB, et al. (1998). State-related changes in upper airway caliber and surrounding soft-tissue structures in normal subjects. Am. J. Respir. Crit. Care Med. 158: 1259-1270.

Tsuiki S, Isono S, Ishikawa T, Yamashiro Y, et al. (2008). Anatomical balance of the upper airway and obstructive sleep apnea. Anesthesiology 108: 1009-1015.

Watanabe T, Isono S, Tanaka A, Tanzawa H, et al. (2002). Contribution of body habitus and craniofacial characteristics to segmental closing pressures of the passive pharynx in patients with sleep-disordered breathing. Am. J. Respir. Crit. Care Med. 165: 260-265.

Worsnop C, Kay A, Pierce R, Kim Y, et al. (1998). Activity of respiratory pump and upper airway muscles during sleep onset. J. Appl. Physiol. 85: 908-920.

Yoshida K, Fukatsu H, Ando Y, Ishigaki T, et al. (1999). Evaluation of sleep apnea syndrome with low-field magnetic resonance fluoroscopy. Eur. Radiol. 9: 1197-1202.

Younes M, Loewen AH, Ostrowski M, Laprairie J, et al. (2012). Genioglossus activity available via non-arousal mechanisms vs. that required for opening the airway in obstructive apnea patients. J. Appl. Physiol. 112: 249-258.

Zhang XY, Yang X, Hua H and Chen JJ (2009). Comparison of MRI fast SPGR single slice scan and continuous dynamic scan in patients with obstructive sleep apnea-hypopnea syndrome. Eur. J. Radiol. 71: 17-21. 